\title{
Croyances, idées reçues et représentations de la maladie cancéreuse
}

\author{
E. Marx $\cdot$ M. Reich \\ (C) Springer-Verlag 2009
}

Pendant des années le cancer a véhiculé une représentation sociale péjorative et taboue. C'était la maladie cachée voire « honteuse », la « peste des temps modernes » et « on n'en parlait pas ».En 2009, cette maladie est devenue moins taboue, et l'on en parle plus facilement. En témoignent les campagnes de dépistage, les informations médiatiques et publicitaires véhiculées par la Ligue contre le cancer et l'INCa. Même si tout est fait sur le plan des campagnes d'information pour démystifier le cancer et lui retirer son image négative (« Nous sommes deux millions de héros ordinaires » une campagne pour faire évoluer l'image du cancer, stipulaient affiches et vidéos de l'INCa en 2007), il reste malgré tout encore synonyme de mort dans l'imaginaire collectif.

L'intensité traumatique du cancer va être modulée tant par des éléments rationnels ou irrationnels intervenant dans l'élaboration d'une psychogenèse de la maladie que par des variables émotionnelles et cognitives. Celles-ci façonnent les comportements et les attitudes face à la maladie, l'adhésion ou pas aux traitements proposés avec le recours possible aux médecines parallèles et à des interprétations parfois totalement irrationnelles.

La découverte d'un cancer va le plus souvent entrâner chez le patient une recherche de sens, soit par interprétation de l'événement ou par attribution d'une signification et la mise en place de croyances, de pensées magiques et de comparaisons sociales.

Ces croyances et représentations véhiculées par le cancer peuvent s'intégrer dans les processus d'adaptation à la maladie. Toutefois, elles ne sont pas l'apanage des seuls malades mais touchent aussi les proches et l'ensemble des professionnels en cancérologie, soignants et non-soignants,

E. Marx $(\bowtie)$

Unité de psycho-oncologie, centre Paul-Strauss,

3, rue de la Porte-de-l'Hôpital, BP 42,

F-67065 Strasbourg cedex, France

e-mail : emarx@strasbourg.fnclcc.fr

M. Reich ( $\square)$

Équipe de psycho-oncologie, centre Oscar-Lambret,

3, rue Frédéric-Combemale, F-59020 Lille, France

e-mail : M-Reich@o-lambret.fr avec des implications non négligeables dans la relation médecin-malade.

Les croyances des soignants concernent les théories causales de la maladie et l'évolutivité attendue (pronostic) basées sur un rationnel «scientifique », le type d'informations délivrées et de soutiens offerts au patient.

Les articles de ce dossier thématique abordent, pour la plupart, cet aspect des croyances du côté des professionnels de santé. Y. Libert et C. Reynaert soulignent dans leur article l'impact potentiel des caractéristiques psychologiques des médecins en particulier du vécu subjectif de contrôle dans la communication et dans la relation qu'ils peuvent avoir avec leurs patients. Le rôle de ces " prédicteurs de l'acquisition de compétences en communication " chez les médecins en termes de croyances internes ou externes sur le contrôle de la qualité de la communication et de la régulation émotionnelle durant l'entretien apparaît comme une piste intéressante dans le champ de la communication en oncologie. A. Desauw et al. explorent quant à eux la perception et le ressenti que peuvent avoir les praticiens confrontés à la délicate mission d'annoncer une mauvaise nouvelle. Là aussi, les médecins, pour éviter d'être débordés par leurs représentations de la maladie qu'ils doivent énoncer à leurs patients, sont amenés à devoir mettre en place selon ces auteurs des « stratégies de régulation émotionnelle ». Cette étude qualitative met en perspective le décalage existant entre les croyances des médecins face aux attentes et représentations de leurs patients et la réalité de ces attentes.

Les croyances et représentations du cancer constituent une interface entre le soignant et le patient dont le thérapeute doit tenir compte. Mais qu'en est-il lorsque le soignant et le patient ne font plus qu'une seule et même personne ? C. Boiron, oncologue médicale, traitée pour un cancer du sein à l'âge de 32 ans, nous livre un témoignage à la fois émouvant et rare. Il n'est en effet pas courant que les professionnels de santé et particulièrement ceux exerçant dans le champ de la cancérologie puissent s'enrichir du vécu d'une des leurs, autorisant ainsi une réflexion sur leur propre pratique professionnelle influencée par leurs représentations et par leurs mécanismes d'adaptation 
psychologiques en œuvre, dès lors qu'ils sont amenés à prendre soin d'un soignant malade du cancer (projection identificatoire, déni, fuite, agressivité, etc.).

Susceptibles d'évolution durant la maladie, les croyances et représentations du cancer sont aussi influencées par les facteurs culturels, sociétaux et philosophiques qui entourent le patient et les soignants en charge de celui-ci.

F. Soum-Pouyalet et al. analysent l'utilisation des termes «moral» ou «bon moral» par les soignants et les représentations qu'ils recouvrent. Objet d'interprétation propre à chaque individu, le « moral », processus d'adaptation, révèle un état fluctuant rendant compte des liens entre le physique et le psychique.
L'article de V. Regnier-Denois et al. aborde, dans le contexte des avancées thérapeutiques en cancérologie, les comportements et les pratiques des oncologues et des patients liés à l'administration de produits de chimiothérapies par voie orale. Là où l'information du patient demeure toujours complexe, ces réactions et ces attitudes font intervenir les représentations de l'efficacité des traitements et la mémoire de la maladie.

C'est donc un des objectifs et non des moindres de ce dossier thématique de la revue Psycho-Oncologie de permettre à différents intervenants, et ce de façon pluridisciplinaire, de livrer au travers de leur vécu, de leur expérience clinique et de recherche, leur vision moderne de ces problématiques complexes. Nous vous en souhaitons bonne lecture ! 